University of Wollongong

Research Online

Faculty of Engineering and Information

Faculty of Engineering and Information

Sciences - Papers: Part A

Sciences

$1-1-2000$

\title{
A geometric approach for three-phase load balancing in distribution networks
}

Kashem M. Muttaqi

Queensland University of Technology, kashem@uow.edu.au

Velappa Ganapathy

Multimedia University

G B. Jasmon

Multimedia University

Follow this and additional works at: https://ro.uow.edu.au/eispapers

Part of the Engineering Commons, and the Science and Technology Studies Commons

Research Online is the open access institutional repository for the University of Wollongong. For further information contact the UOW Library: research-pubs@uow.edu.au 


\section{A geometric approach for three-phase load balancing in distribution networks}

\section{Keywords}

networks, load, distribution, phase, three, approach, geometric, balancing

Disciplines

Engineering | Science and Technology Studies

\section{Publication Details}

M. A. Kashem, V. Ganapathy \& G. B. Jasmon, "A geometric approach for three-phase load balancing in distribution networks," in IEEE-PES/CSEE International Conference on Power System Technology (PowerCon 2000), 2000, 


\section{A GEOMETRIC APPROACH FOR THREE-PHASE LOAD BALANCING IN DISTRIBUTION NETWORKS}

\author{
M. A. Kashem \\ Electrical and Electronic Systems Engineering \\ Queensland University of Technology \\ Brisbane, Queensland, Australia
}

\author{
V. Ganapathy, G. B. Jasmon \\ Multimedia University \\ Cyberjaya, Selangor, \\ Malaysia
}

\begin{abstract}
Network reconfiguration for load balancing is the determination of switching-options for a particular set of loads on a distribution system that relieves overloads and improves load balancing. It is performed by altering the topological structure of distribution feeders which is done by changing the open / closed status of sectionalizing and tie-switches. To relieve overloads of a network and to balance feeder loads, the loads are transferred from the heavily loaded feeders to the lightly loaded ones. In this paper, a geometric approach for load balancing is presented. In the proposed method, each loop in a network is represented as a circle, which is derived from the relationship between the change of load balancing due to the branch-exchange and the powerflows in the branches. If there is no change of load balancing in the system, then all the circles touch each other at the $(0,0)$ coordinate. The circles with no load balancing are called zero load balancing change circles. The maximum load balancing loop in the network is identified by comparing the radii of all the modified zero load balancing change circles. The corresponding loop of the largest one gives the maximum improvement of load balancing in the network. Then the possible branch exchanges in the maximum load balancing loop are investigated by comparing the size of the circle for every branch-exchange. If the loads are balanced due to a branch exchange, the size of the circle diminishes and hence the smallest circle gives the maximum improvement in load balancing and the corresponding branchexchange is considered to be the best candidate for maximum improvement in load balancing. To show the efficiency and performance of the proposed method for the solution of computationally complex and large dimensionality problems, a system with 69-bus and 7 laterals has been considered as the test system. Test results have shown that the proposed method can identify the most effective branch-exchange operations for load balancing with less computational effort and time. The number of load flow solutions has been reduced to a greater extent in the proposed method.
\end{abstract}

Keywords: Distribution networks, Load balancing, Geometric, and Network reconfiguration.

\section{INTRODUCTION}

The increase in power demand and high load density in the urban areas makes the operation of distribution systems more complex to control. To meet the load demand, the requirements are met by increasing the substation capacity and the number of feeders. Due to financial and other constraints the requirements could not be met. Therefore, load-balancing techniques are resorted to provide more capacity margin for the substation to meet the extra load demand. With the advent of fast computing systems, the advanced control of electrical power systems has become a viable one. For effective coordination of their protective systems, the distribution systems are normally configured radially. Most distribution networks use sectionalizingswitches that are normally closed, and tie-switches that are normally opened. These switches are used for both protection and network reconfiguration. Due to changing operating conditions, networks are reconfigured to reduce the system power loss i.e., network reconfiguration for loss reduction, and to relieve overloads in the networks i.e., network reconfiguration for load balancing. By modifying the radial structure of the feeders periodically may significantly improve the operating conditions of the overall system. Feeders in a distribution system normally have a mixture of industrial, commercial, residential, lighting etc. type of loads. The peak load on the substation transformers and feeders occur at different times of the day. Therefore, the distribution system becomes heavily loaded at certain times of the day, and lightly loaded at other times. Load balancing is obtained by transferring loads from the heavily loaded feeders to lightly loaded feeders by reconfiguring the network. This is done in order to reschedule the loads more efficiently for maximizing the load balancing in the system. Reconfiguration also allows smoothening out the peak demands, improving the voltage profile in the feeders and increasing the network reliability.

Aoki et. al. [1,2] have formulated the load balancing and service restoration problems by considering the capacity and voltage constraints as a mixed integer non-linear optimization problem. Baran et. al. [3] have formulated the problem of loss minimization and load balancing as an integer-programming problem. Chiang et. al. [4] have proposed a constrained multi-objective and non- 
differentiable optimization problem, with equality and inequality constraints for both loss reduction and load balancing. Peponis et. al. [5] have developed an improved switch-exchange method for load balancing problem using switch exchange operations. Kashem et. al. [6] have proposed a new load balancing index and applied it to the network for load balancing. It has been shown in [6] that improvement in load balancing can be achieved by network reconfiguration. Lin et. al. [8] have presented a new load balancing algorithm for the three-phase unbalanced distribution systems. The proposed algorithm of [8] could confine the size of the solution state-space, and avoid complex numerical computations. M. A. Kashem et.al. [9] have developed a technique for load balancing by measuring the distances of various points representing power-flows.

A new approach for network reconfiguration based load balancing is presented in this paper by introducing a geometrical technique. The method can be implemented by measuring and comparing the radii of the circles, which are formed from the relation of power-flows in the branches. The first stage of the method determines the maximum load-balancing loop graphically. In a distribution system a loop is associated by a tie-line and hence there are several loops in the system. To obtain the maximum loadbalancing loop, the zero load balancing-change circles are drawn and the loop with the largest circle is identified for maximum load balancing. The second stage determines the switching-operation to be executed in that loop to reach a maximum improvement in load balancing by network configuration. The smallest circle is identified for the best solution, as the size of the loop circle is reduced when the load balancing is improved. By utilizing this proposed geometrical technique, an optimal or near optimal configuration for load balancing can be obtained with reduced computational effort because it does not have to check the branches in all the loops to obtain maximum improvement in load balancing as used in reference 3 . The proposed method will be a useful tool for real-time control of automated distribution system for load balancing.

\section{GEOMETRICAL APPROACH FOR LOAD BALANCING DUE TO A BRANCH EXCHANGE}

A radial distribution network can be represented by several loops. The number of loops is equal to the number of tielines because one tie-line can only make one loop when it is connected. A loop in a radial network is created by a tieline $t$ as shown in Figure 1. There are two sides in a loop, one is the lower voltage side and the other is the higher voltage side denoted by $l$ and $h$ respectively.

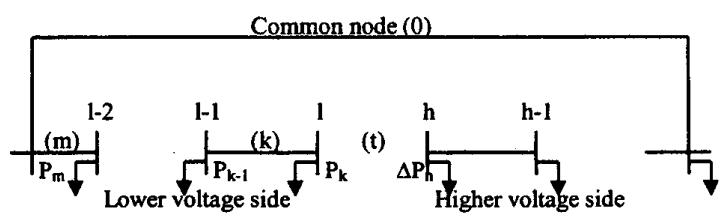

Figure 1: The loop associated with tie-line $t$

Consider the branch exchange between branches $t$ and $m$ where $t$ is the open branch and $m$ is the closed branch in Fig.1. The loop is divided into two parts by the open branch $t$ in which the first part consisting the branches in the loop that extends between nodes 0 to $l$ and the second part consisting the branches that extends between nodes 0 to $h$. The change in load balancing formula due to this branch exchange as given in reference 9 is rewritten as follows:-

$$
\begin{aligned}
& \Delta \mathrm{LB}_{\mathrm{tm}}^{\mathrm{S}}=2 \mathrm{P}_{\mathrm{m}}\left(\sum \mathrm{k}_{1} \mathrm{P}_{1}-\sum \mathrm{k}_{\mathrm{h}} \mathrm{P}_{\mathrm{h}}\right)+2 \mathrm{Q}_{\mathrm{m}}\left(\sum \mathrm{k}_{\mathrm{l}} \mathrm{Q}_{\mathrm{l}}-\right. \\
& \left.\sum \mathrm{k}_{\mathrm{h}} \mathrm{Q}_{\mathrm{h}}\right)-\left(\mathrm{P}_{\mathrm{m}}{ }^{2}+\mathrm{Q}_{\mathrm{m}}{ }^{2}\right) \mathrm{k}_{\text {loop }}
\end{aligned}
$$

where,

$\Delta \mathrm{LB}_{\mathrm{tm}}^{\mathrm{S}}$ : the change in load balancing due to branch exchange $t-m$,

$P_{\mathrm{in}}$ and $\mathrm{Q}_{\mathrm{m}}$ : real and reactive power flows in branch $m$ (to be removed) respectively,

$\mathrm{k}_{i}$ : the inverse of the square of the maximum capacity of a branch $i$,

$\mathrm{k}_{\mathrm{l}} \mathrm{P}_{\mathrm{t}}$ and $\mathrm{k}_{\mathrm{h}} \mathrm{P}_{\mathrm{h}}$ : products of $k$ and $P$ on the lv-side $l$ and hvside $h$ of the loop respectively,

$\mathrm{k}_{\text {loop }}$ : the sum of $k$ value of each branch in the loop in which power-flow exists.

If the value of $\Delta \mathrm{LB}_{\mathrm{tm}}^{\mathrm{S}}$ is greater than zero, the load balancing is improved, and if it is less than zero, the load balancing is deteriorated.

Equation (1) can be rearranged as shown below to be represented by a circle,

$\left[\mathrm{P}_{\mathrm{m}}-\mathrm{A} / \mathrm{C}\right]^{2}+\left[\mathrm{Q}_{\mathrm{m}}-\mathrm{B} / \mathrm{C}\right]^{2}=[\mathrm{A} / \mathrm{C}]^{2}+[\mathrm{B} / \mathrm{C}]^{2}-\Delta \mathrm{LB}_{\mathrm{tm}}^{\mathrm{S}} / \mathrm{C}(2)$ where, $A=\sum k_{1} P_{1}-\sum k_{h} P_{b}, \quad B=\sum k_{1} Q_{1}-$ $\sum \mathrm{k}_{\mathrm{h}} \mathrm{Q}_{\mathrm{h}}$ and $\mathrm{C}=\mathrm{k}_{\text {loop }}$

The center of the circle is $(\mathrm{A} / \mathrm{C}, \mathrm{B} / \mathrm{C})$ and its radius is $\left[\frac{A^{2}+B^{2}}{C^{2}}-\frac{\Delta L B_{i m}^{s}}{C}\right]^{\frac{1}{2}}$.

The zero load balancing change circle is the one which gives the change in load balancing to be zero (i.e. $\Delta \mathrm{LB}_{\mathrm{tm}}^{\mathrm{S}}=$ $0)$. It can be represented as follows:

$\left[\mathrm{P}_{\mathrm{m}}-\mathrm{A} / \mathrm{C}\right]^{2}+\left[\mathrm{Q}_{\mathrm{m}}-\mathrm{B} / \mathrm{C}\right]^{2}=[\mathrm{A} / \mathrm{C}]^{2}+[\mathrm{B} / \mathrm{C}]^{2}$

Hence the radius of the circle is $\left[\frac{A^{2}+B^{2}}{C^{2}}\right]^{\frac{1}{2}}$ for zero load

balancing change (i.e. $\Delta \mathbf{L B} \mathbf{t m}_{\mathrm{tm}}^{\mathrm{S}}=0$ ) and is decreased if load balancing is improved in the loop. 


\section{II.A GEOMETRICAL DETERMINATION OF THE MAXIMUM LOAD BALANCING LOOP}

The basic idea of the proposed search scheme is by first identifying the maximum load balancing loop in the network and then a switching-option is determined for the loop that gives a maximum improvement in load balancing.

Equation (2) can be rearranged as,

$\Delta \mathrm{LB}_{\mathrm{tm}}^{\mathrm{S}}=\left(\mathrm{A}^{2}+\mathrm{B}^{2}\right) / \mathrm{C}-\mathrm{C}\left\{\left(\mathrm{P}_{\mathrm{m}}-\mathrm{A} / \mathrm{C}\right)^{2}+\left(\mathrm{Q}_{\mathrm{m}}-\mathrm{B} / \mathrm{C}\right)^{2}\right\}$

From equation (4), it is evident that the change in load balancing, $\Delta \mathrm{LB}_{\mathrm{tm}}^{\mathrm{S}}$ will be maximum when $\mathrm{P}_{\mathrm{m}}=\mathrm{A} / \mathrm{C}$ and $\mathrm{Q}_{\mathrm{m}}=\mathrm{B} / \mathrm{C}$. Therefore, the maximum value of $\Delta \mathbf{L} \mathbf{B}_{\mathrm{tm}}^{\mathrm{S}}$ in a loop is

$\Delta \mathrm{LB}_{\text {tm loop }}^{\mathrm{s}_{\text {lop }}}=\left(\mathrm{A}^{2}+\mathrm{B}^{2}\right) / \mathrm{C}$

The equation for $\Delta L B_{\text {tm loop }}^{S}$ is similar to the radius of the zero load balancing change circle except the constant of multiplication in the equation.

Rearranging equation (3), we get the modified zero-load balancing change circle as,

$\left[P_{m}^{\prime}-\frac{A}{\sqrt{C}}\right]^{2}+\left[Q_{m}^{\prime}-\frac{B}{\sqrt{C}}\right]^{2}=\frac{A^{2}+B^{2}}{C}$

where, $P_{m}^{\prime}=\sqrt{C} P_{m}$ and $Q_{m}^{\prime}=\sqrt{C} Q_{m}$

The radius of the above circle is $\left[\frac{A^{2}+B^{2}}{C}\right]^{\frac{1}{2}}$ which is the

square root of $\Delta \mathrm{LB}_{\mathrm{tm}}^{\mathrm{s}}$ value. The loop circles can be drawn using equation (6) and the largest circle will give the maximum loss reduction loop among all the circles drawn for all the loops in the network.

The drawback of equation (5) is that the value of the

$\Delta \mathrm{LB}_{\text {tm loop }}^{\mathrm{S}}$ is always a positive constant for a particular loop as it is independent of $P_{m}$ and $Q_{m}$. However, load balancing depends on the value of $\Delta \mathrm{LB}_{\mathrm{tm}}^{\mathrm{S}}$ i.e. if it is tve, load balancing is improved and if it is -ve, load balancing is deteriorated [9]. Whether the load balancing is improved or deteriorated cannot be shown by equation (5) because it is always positive. Therefore to ensure that the largest circle gives the maximum improvement in load balancing, the nominal branch is considered. The nominal branch is the first adjacent branch to the tie branch on the lower voltage side of the loop and the nominal load balancing is the load balancing which occurs by exchanging the open branch with the nominal branch. If the nominal load balancing is negative, then there is no branch in the loop that can be a candidate for branch exchange [9]. If the nominal load balancing is positive, it means that maximum load balancing is achieved and the branch in the loop for branch exchange is determined.
Equation (4) can be rewritten for nominal branch exchange as,

$\Delta \mathrm{LB}_{\mathrm{tk}}^{\mathrm{S}}=\left(\mathrm{A}^{2}+\mathrm{B}^{2}\right) / \mathrm{C}-\mathrm{C}\left\{\left(\mathrm{P}_{\mathrm{k}}-\mathrm{A} / \mathrm{C}\right)^{2}+\left(\mathrm{Q}_{\mathrm{k}}-\mathrm{B} / \mathrm{C}\right)^{2}\right\}$ where, $P_{k}$ and $Q_{k}$ are the power flows in the nominal branch k.

Using equation (7) the nominal loop circle is drawn for nominal branch exchange and compared with the zero load balancing change circle drawn by using equation (3) in the respective loop. If the nominal loop circle is reduced in size, then the nominal load balancing is positive and the branch exchange in the loop gives the maximum load balancing. Otherwise the next largest circle is considered and checked as above.

\section{II.B GEOMETRICAL DETERMINATION OF SWITCHING-OPTION FOR LOAD BALANCING}

In network reconfiguration, multiple switching-options are possible for optimal or near optimal configuration where several tie and sectionalizing switches are simultaneously closed and / or opened by the successive application of the proposed scheme. The best switching-option to be implemented is chosen in each successive operation that maximizes load balancing the most, without violating the constraints such as voltage, capacity of lines and transformers and reliability.

The authors in [9] have established a rule for the switching exchange of switches by considering the lower and higher voltage side of the loop. In ref. 9, load balancing is achieved when loads are transferred from the lower voltage side of a loop to the higher voltage side of that loop. It was noted that each line contains a sectionalizing switch to be operated for network reconfiguration. In this paper, branches of lower voltage side of a loop are considered for branch exchange and the most suitable switching-exchange is found by the knowledge of geometry as described below.

After determining the loop that would give the maximum load balancing by the geometrical procedure described in the previous section, a branch-exchange is identified in that loop. The various circles are drawn by using equation (4) for all the branches in the lower voltage side of the loop. The size of the circle depends on the value of the $\Delta \mathrm{LB}_{\mathrm{tm}}^{\mathrm{S}}$. It gets reduced as the $\Delta \mathrm{LB}_{\mathrm{tm}}^{\mathrm{S}}$ value is positive and increased if $\Delta \mathrm{LB}_{\mathrm{tm}}^{\mathrm{S}}$ is negative. When $\Delta \mathrm{LB}^{\mathrm{S}}{ }_{\mathrm{tm}}$ is positive maximum for a branch-exchange, then the corresponding circle will be the smallest one. Therefore, the smallest circle will give the best solution and the corresponding branch-exchange will give the maximum load balancing in the network. 


\section{SOLUTION TECHNIQUE}

The procedure to determine the loop, which gives the maximum improvement in load balancing, is summarized below.

i) Run the load flow program to obtain the estimates of the power flows in the branches.

Determine the radii of the modified zero-load balancing change circles for all the loops in the system (equation (6)) and compare. Select the largest circle and the corresponding loop.

Check the nominal branch-exchange in the selected loop whether it contributes positive load balancing or not. This is done by comparing the radius of the nominal loop circle (equation (7)) with the radius of zero-load balancing change circle (equation (3)). If nominal loop circle is reduced in size, then go to step (v). Otherwise select the next largest circle and repeat step (iii). If all the nominal loop circles are increased in size compared to their respective zero-load balancing change circles, then stop.

v) Identify the loop of the corresponding circle and declare it as the maximum load balancing loop.

After finding the loop that gives the maximum load balancing, the next step is to determine the branch to be exchanged.

vi) Start from the nominal branch of the selected loop in step (v) and search the branches backward on the lower voltage side of the loop until the radius of the loop circle (equation (4)) is found to be minimum. Identify the corresponding branchexchange.

vii) Check the selected branch-exchange for constraints violation. If all the constraints are satisfied, go to step (viii); otherwise discard the selected branch-exchange and continue the search forward and backward (one by one), until the constraints are satisfied and also the radius is found to be minimum.

viii) Declare the selected branch-exchange for maximum load balancing.

ix) Repeat steps (i) to (viii) using the selected branchexchange.

\section{TEST RESULTS AND DISCUSSIONS}

The test system is a hypothetical $12.66 \mathrm{kV}$ radial distribution system with 69 buses and 7 laterals, which has been derived from a portion of the PG\&E distribution system [7]. The schematic diagram of the test system is shown in Figure 2 and the relevant data are same as in ref. 7. There are 5 tie-lines (looping branches) in the system and sectionalizing switches on every branch of the system.
The total system loads for the base configuration are $3802.19 \mathrm{~kW}$ and $2694.60 \mathrm{kVAr}$.

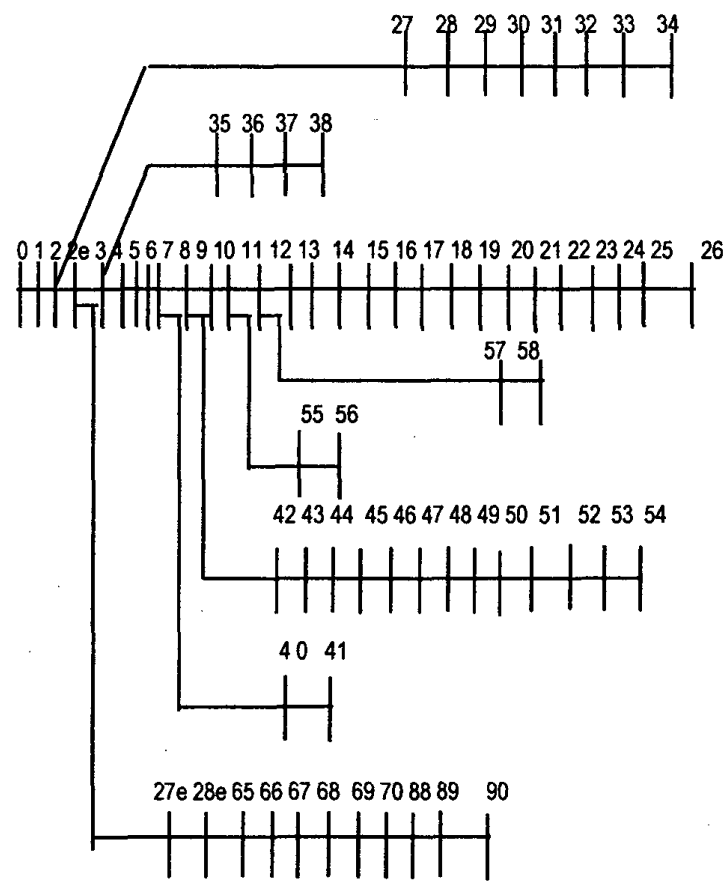

Fig. 2: Schematic diagram of a 69-bus distribution system.

The proposed geometrical method is tested on the 69-bus test system and test results are given in Table 1. It is shown that at the first search-level, at which, the radii of modified zero-load balancing change circles for all loops are calculated, and the loop that gives the largest value of radius is selected. In the next step, a comparison is made between the nominal loop circle and the zero-load balancing change loop circle to make sure that the selected loop gives the improvement in load balancing. The nominal circle has to be smaller to achieve load balancing in the system. In the next stage, the branch-exchange in that loop is selected by determining and comparing the radii of the loop circles starting from the nominal branch and searching the branches backward in the lower voltage side of the selected loop until the smallest circle is found to give the maximum improvement in load balancing. As shown in Table 1, the first iteration of the proposed method has found loop 4 as the maximum load balancing loop, and the branch exchange $73-59$ to be performed in that loop to achieve maximum load balancing as shown in Figures 3 and 4 respectively. In Figure 3, the largest circle is found as the circle for the loop 4, which gives the maximum loadbalancing loop. The loop circles are drawn for all the branch-exchanges in the lower voltage side of the loop 4 in Figure 4(a). It is visualized that the smallest circle is the circle for branch-exchange 73-59. In Figure 4(b) five small circles are shown and the smallest one is the circle for the 
branch-exchange 73-59, which gives the maximum load balancing. The above procedure is continued until all the loops have the nominal circles larger compared to their respective zero-load balancing change circles. It is to be noted that there is no need to draw the loop circles and the decision can be taken by comparing the radii of the circles only.

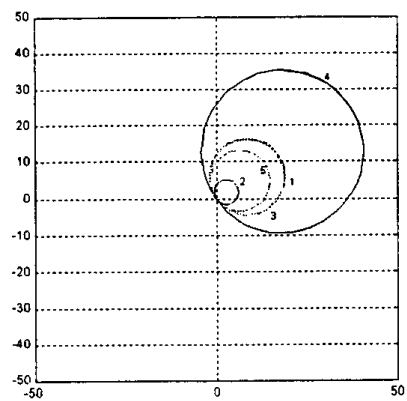

Figure 3: Maximum load balancing loop 4 has been selected in the first search

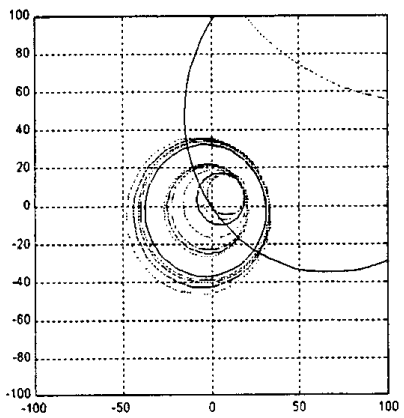

Figure 4(a): Circles for all the branch-exchanges in the lower voltage side of loop 4

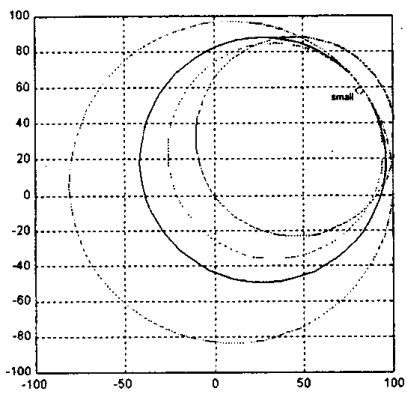

Figure 4(b): 5 small circles are shown and Branch-exchange 73-59 has been identified

Table 2 shows the summary of the selection of branchexchanges for load balancing in all the search-levels. The optimal network configuration for load balancing (shown in Figure 5) is achieved after 5 search-levels of the proposed method, in which, at each search level (or iteration) a load flow solution is obtained, the maximum load balancing loop is selected and a branch-exchange is determined. In the fifth search-level, all the nominal circles become larger in size compared to their respective zeroload balancing change circles and these are shown in Table 2.

Table 1: Selection of Brach-Exchange in the First Search-Level of the Proposed Method

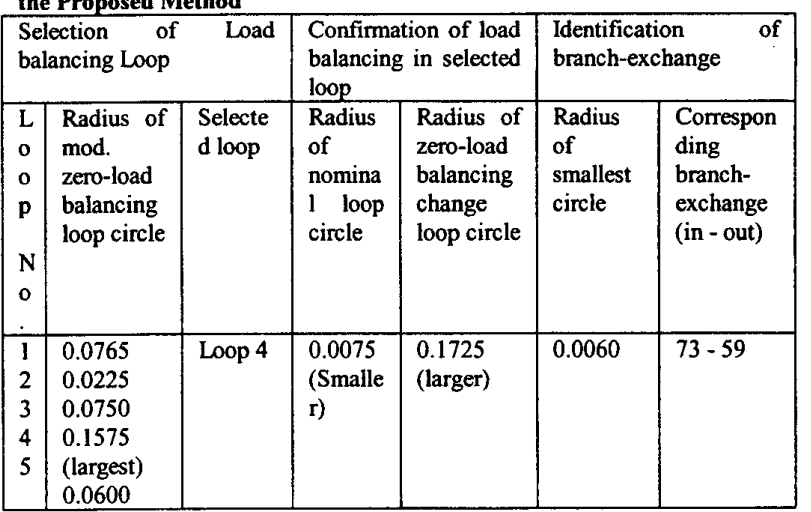

Note: Radius value is in per unit.

The proposed method considers the branches in the loop, which gives maximum improvement in load balancing by considering one branch-exchange in each search level. Because of lesser number of load flow solutions it is seen that the proposed method has greatly reduced computational time compared to Baran and Wu's method [3]. It is experimentally proven that the methods involved with a large number of load flow solutions, some times, seem exhaustive and unrealistic specially for larger systems, as they require more computational time.

Table 2: Selection of Branch-Exchanges by the Proposed Geometrical Method

\begin{tabular}{|c|c|c|c|c|c|c|}
\hline \multirow[t]{2}{*}{$\begin{array}{l}\text { S } \\
\text { ea }\end{array}$} & \multicolumn{2}{|c|}{ Loop Selection } & \multicolumn{2}{|c|}{$\begin{array}{l}\text { Confirmation of load } \\
\text { balancing in selected } \\
\text { loop }\end{array}$} & \multicolumn{2}{|c|}{$\begin{array}{l}\text { Identification } \\
\text { branch-exchange }\end{array}$} \\
\hline & $\begin{array}{l}\text { Radius of } \\
\text { mod. } \\
\text { zero-load } \\
\text { balancing } \\
\text { loop circle }\end{array}$ & $\begin{array}{l}\text { Sele } \\
\text { cted } \\
\text { loop } \\
*\end{array}$ & $\begin{array}{l}\text { Radius of } \\
\text { nominal } \\
\text { loop circle }\end{array}$ & $\begin{array}{l}\text { Radius of } \\
\text { zero-load } \\
\text { balancing } \\
\text { change } \\
\text { loop circle }\end{array}$ & $\begin{array}{l}\text { Radius } \\
\text { of } \\
\text { smallest } \\
\text { circle }\end{array}$ & $\begin{array}{l}\text { Correspon } \\
\text { ding } \\
\text { branch- } \\
\text { exchange } \\
\text { (in - out) }\end{array}$ \\
\hline 1 & 0.1575 & 4 & 0.0075 & 0.1725 & 0.0060 & $73-59$ \\
\hline 2 & 0.1275 & 3 & 0.0270 & 0.0870 & 0.0180 & $72-13$ \\
\hline 3 & 0.2370 & 5 & 0.0900 & 0.1500 & 0.0975 & $74-62$ \\
\hline 4 & 0.1425 & 3 & 0.0225 & 0.0975 & 0.0075 & $13-15$ \\
\hline 5 & $\begin{array}{l}0.4470 \\
0.4395 \\
0.0750 \\
0.0300 \\
0.0045\end{array}$ & $\begin{array}{l}1 \\
2 \\
3 \\
4 \\
5\end{array}$ & $\begin{array}{l}0.0750 \\
0.2475 \\
0.0945 \\
0.1275 \\
0.1200 \\
\end{array}$ & $\begin{array}{l}0.0225 \\
0.0060 \\
0.0645 \\
0.0900 \\
0.0975\end{array}$ & \multicolumn{2}{|c|}{$\begin{array}{l}\text { No Loop is found to } \\
\text { give load balancing. }\end{array}$} \\
\hline
\end{tabular}

Note: Radius value is in per unit.

'(in - out)' indicates branch into the system and branch out from the system respectively.

* Loopl is associated with tie-line 70, loop2 with tie-line 71 , loop3 with tie-line 72, loop4 with tie-line 73 and loop5 with tie-line 74. 


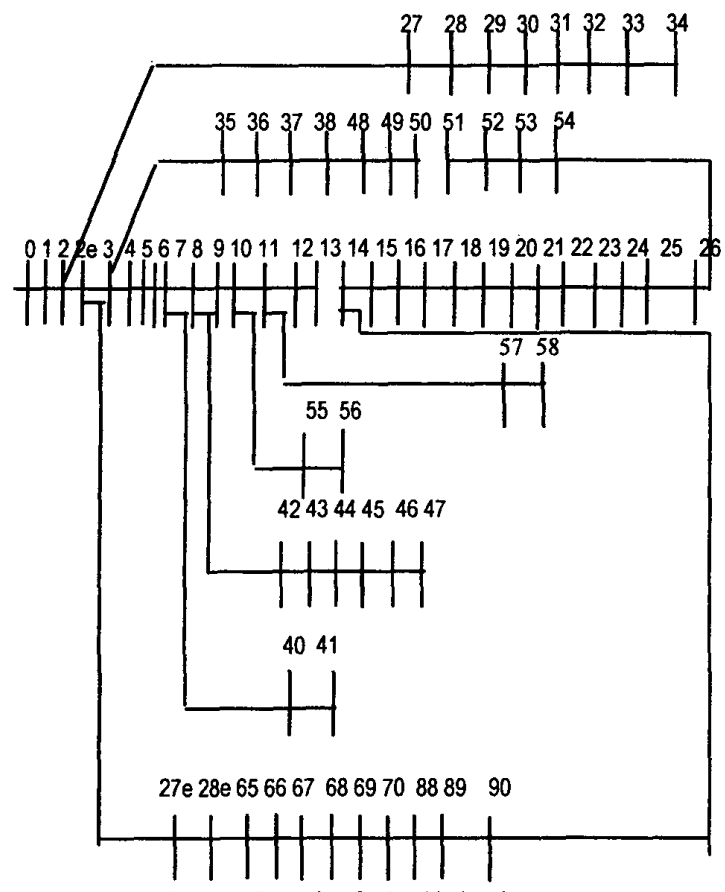

Fig. 5: The optimal configuration for load balancing

\section{CONCLUSION}

A geometrical approach for load balancing has been presented in this paper. In the proposed method, various circles, which are formed from the power flows in the branches, are compared. A two-stage searching approach to the switching operation is proposed. In the first stage, the modified zero-load balancing circles are compared for various loops and the largest one is selected as the maximum load balancing loop. To ensure the load balancing for a branch-exchange in the selected loop, the nominal loop circle is compared with the zero-load balancing change circle. If the nominal loop circle-size is reduced, then the corresponding loop gives the maximum load balancing. Otherwise the next largest circle is considered. In the second stage, the circles for various branch-exchanges in the selected loop are compared and the smallest one is identified for the best solution. The corresponding branch-exchange of the smallest circle gives the maximum load balancing in the system. The proposed method has been applied to a 69-bus test system and the improvement in efficiency and the performance are established. From the test results it is found that the proposed method has identified the most effective branchexchange operations for load balancing.

\section{REFERENCES}

1. K. Aoki, H. Kuwabara, T. Satoh, and M. Kanezashi, 'An Efficient Algorithm for Load Balancing of Transformers and Feeders by Switch Operation in Large Scale Distribution Systems', IEEE PES Summer Meeting, 1987, Paper no.: 87SM 543-2.

2. K. Aoki, T. Satoh, M. Itoh et. al., 'Voltage Drop Constrained Restoration of Supply by Switch Operation in Distribution Systems', IEEE PES Summer Meeting, 1987, Paper no.: 87SM5440 .

3. M. E. Baran, F. F. Wu, Network Reconfiguration in Distribution Systems for Loss Reduction and Load Balancing', IEEE Trans. on Power Delivery, Vol.4, No.2, April 1989, pp.1401-1407.

4. H. D. Chiang and R. J. Jumeau, 'Optimal Network Reconfigurations in Distribution Systems: Partl : A New Formulation and A Solution Methodology', IEEE Trans. on Power Delivery, Vol.5, No.4, Nov.1990, pp.1902-1909.

5. G. Peponis, M. Papadopoulos, 'Application of Heuristic Methods on Large-scale Networks', IEE Proc.-Gener. Transm. Distrib., Vol.142, No. 6, Nov.1995, pp.631-638.

6. M. A. Kashem and M. Moghavvemi, 'Maximizing Radial Voltages Stability and Load Balancing via Loss Minimization in Distribution Networks', Proc. of the International Conference on Energy Management and Power Delivery (EMPD'98), Singapore, 3-5 March 1998.

7. Mesut E. Baran and Felix F. Wu, 'Optimal Capacitor Placement on Radial Distribution Systems', IEEE Trans. on Power Delivery, Vol.4, No.1, Jan. 1989, pp.725-734.

8. Whei-Min Lin, Hong-Chan Chin, 'A Current Index Based Load Balancing Technique for Distribution Systems', Proceedings of International Conference on Power System Technology, 1998, (POWERCON'98), Vol.1, pp.223-227.

9. M. A. Kashem, V. Ganapathy, and G. B. Jasmon, "Network Reconfiguration for Load Balancing in Distribution Networks", IEE Proceedings - Generation, Transmission and Distribution, Vol.146, No.6, Nov.1999, pp.563-567. 